\title{
GLOBAL AND LOCAL LINEAR BUCKLING BEHAVIOR OF A CHIRAL CELLULAR STRUCTURE
}

\author{
Alessandro Spadoni, Massimo Ruzzene \\ School of Aerospace Engineering \\ Georgia Institute of Technology \\ Atlanta, GA 30332 \\ Fabrizio Scarpa \\ Department of Mechanical Engineering \\ University of Sheffield \\ Sheffield, UK
}




\begin{abstract}
This paper investigates the flat-wise compression behavior of an innovative cellular structure configuration. The considered layout has a hexagonal chiral geometry featuring cylinders, or nodes, joined by ligaments, or ribs. The resulting assembly is characterized by a number of interesting properties that can be exploited for the design of alternative honeycombs or cellular topologies to be used in sandwich construction. The flat-wise strength of the chiral geometry is investigated through classical analytical formulas for the linear buckling of thin plates and shells and a bifurcation analysis performed on a Finite Element model. The analytical expressions predict the global buckling behavior and the resulting critical loads, and can be directly compared with the results obtained from the Finite Element analysis. In addition, the Finite Element model also predicts local buckling modes, which should be considered to evaluate the possible development of localized plasticity. A sensitivity study is performed to evaluate the influence of the geometry of the chiral structure on its buckling strength. The study shows that the considered topology can offer great design flexibility, whereby several parameters can be selected and modified to improve the flat-wise performance. The comparison with traditional, hexagonal centro-symmetric structural configurations concludes the paper and demonstrates the enhanced performance and the potentials of chiral noncentrosymmetric designs.
\end{abstract}




\section{INTRODUCTION}

The multifunctional properties of cellular solids have generated great interest for their application in ultra-light structures. Their multi-physics characteristics are highly dependent on the architecture of the cells composing the material, which can be designed to enhance simultaneously mechanical, electromagnetic and thermal performances. The application of stronger and lighter structural assemblies and materials is particularly relevant to the aerospace and aircraft industries. Ongoing interests in such structures and improvements in manufacturing processes have contributed to the study and development of cellular solids of innovative shapes and topologies. Much interest has recently centered on cellular assemblies featuring a negative Poisson's ratio behavior, also known as "auxetic" [1]. Materials having auxetic characteristics include special subsets of foams [2], long fiber composites [3], microporous polymers [1], as well as honeycombs [4]. In honeycombs, the negative Poisson's ratio behavior implies a stiffening geometric effect, which leads to increased in-plane indentation resistance, shear modulus and compressive strength $[4,5]$. The auxetic behavior also leads to a synclastic curvature feature, which is extremely useful in manufacturing curved sandwich shells [6].

This work takes into account an alternative and innovative configuration for cellular structures. The considered architecture has chiral geometry and was originally proposed by Prall and Lakes [7]. It is composed of circular elements (nodes) of equal radii joined by straight ligaments (ribs), which are tangent to the nodes (Figure 1). This configuration provides the cellular assembly with distinctive mechanical properties, such as high shear rigidity, negative Poisson's ratio, and a deformation mechanism allowing high strains in the elastic range of the constitutive material. Herein this work, the authors aim at investigating the out-of-plane buckling behavior of a chiral assembly to estimate the potentials for its application as an alternative honeycomb design and to assess its strength under flat-wise compression (Figure 2). The out-of-plane properties of traditional honeycombs have been widely investigated in studies by Gibson and Ashby [8] and Zhang and Ashby [9], among others. On the premises of the work presented by Prall and Lakes [7], Gibson and Ashby [8], and Zhang and Ashby [9], the out-of-plane uniaxial loading of chiral honeycombs (see Figure 2) is here analyzed through analytical formulas 
and a linear buckling analysis carried out using the Finite Element (FE) package ANSYS $^{\circledR}[10]$ The paper is organized in four sections. In section 1, a brief introduction is given. Section 2 describes the objectives of the work and the configuration of the considered geometry, in addition to the FE model and the analytical formulas used in the study. Section 3 discusses results and comparisons with hexagonal structures. Finally, Section 4 summarizes the main results of the work and comments on their implications.

\section{FINITE ELEMENT MODEL OF A CHIRAL CELLULAR STRUCTURE}

\subsection{Overview and objectives}

The flat-wise compression of a chiral honeycomb assembly is investigated through a linear elastic explicit FE model, which is developed to evaluate the critical buckling loads of the considered cellular architecture. Linear buckling has in fact been identified $[8,9]$ as one of the failure mechanisms for traditional hexagonal honeycombs under flat-wise compression.

\subsection{Geometry and configuration}

The chiral honeycomb consists of cylinders or nodes that are connected by straight walls or ribs (Figure 1). The distance between the centers of adjacent nodes is denoted as $R$, while $L$ and $r$ denote respectively the length of the ribs, and the radius of the nodes. The angle $\theta$ characterizes the configuration of the chiral assembly, and is equal to $30^{\circ}$ for a hexagonal configuration. The angle $\beta$ describes the orientation of the ribs with respect 
to the line joining the center of the nodes. Finally, the thickness of the node and rib walls is described by the parameter $t$. The following relationships hold [7]:

$$
\sin \beta=\frac{2 r}{R}, \tan \beta=\frac{2 r}{L}
$$

\subsection{Unit cell identification}

The analysis of the proposed architecture takes advantage of the structure's spatial periodicity to reduce the computational effort, and to minimize the influence of end effects on the estimation of the critical buckling loads. The present study is performed by considering an infinite assembly whose behavior is defined by a unit cell connected to its neighboring cells through periodic conditions. The representative cell is selected as indicated by Papka and Kyriakides [11] and it is shown in Figure 3. The unit cell must possess the same geometry and degrees of freedom of the multicell honeycomb structure to simulate the modes of instability encountered by the entire assembly, and must be able to reproduce the main mechanisms of collapse. Both requirements are satisfied by the unit cell depicted in Figure 3. The cell consists of a central node from which radial ribs expand to the perimeter nodes. The perimeter ribs connecting the perimeter nodes are a distinct feature of chiral honeycombs, and therefore need to be included in the unit cell. They in fact affect the buckling response of the structure and their contribution is essential to comply with the first requirement for a unit cell [11]. An obvious final requirement is that the repeating the cell replicates a multi-cell layout, such as the one shown in Figure 1.a. The perimeter nodes hence, have to be halved to achieve the ability of replicating a unit cell and obtain the complete assembly.

\subsection{Mesh and Element Description}

In the FE model, the semicircular lines constituting the halved-perimeter nodes are divided into six segments, while the straight lines constituting the ribs are divided into 
five elements, as shown in Figure 4.a. The element chosen for the FE model is the eightnodeSHELL93 in ANSYS ${ }^{\circledR}$, with Hermite interpolation functions. This element features 6 degrees of freedom at each node describing translations and rotations in the $x, y$, and $z$ directions. The shape functions are quadratic in both in-plane directions, $x$ and $y$. In addition, the element features plasticity, stress stiffening, large deflection, and large strain capabilities. The mesh for the model is composed of quadrilateral elements, as shown in Figure 4.b.

\subsection{Boundary and Symmetry Conditions}

In order to accurately portrait the buckling mechanism and reproduce conditions similar to flat-wise compression tests, the nodes on the upper edge of the unit cell are left completely unconstrained, while the displacements in the $x, y$, and $z$ directions of the nodes on the lower edge are constrained to zero. Furthermore, periodic boundary conditions relating displacements and rotations of the nodes on the cell perimeter are imposed to simulate the behavior of the cell as part of an infinite assembly. Figure 5 provides a graphical representation of the relationships imposed between the end nodes. Each node belonging to the cell boundary is constrained to move identically to its symmetry mate both in terms of displacements, and in terms of rotations, thereby simulating an infinite structure [11].

\subsection{Applied Loads and Eigenvalue Analysis}

The unit cell is loaded by a downward unit force along the $z$ axis. The applied load is distributed on the nodes belonging to the upper edge as shown in Figure 6. As described in Section 2.4, the element employed for the linear bifurcation analysis is a thin shell element. According to the classical approach to buckling analysis using the FE method [12], the stress, or geometric, stiffness matrix $\left[K_{\sigma}\right]$ required to account for membrane stresses is evaluated through a pre-stress solution pass. Specifically, a reference load is applied to evaluate the corresponding membrane forces $N_{x}, N_{y}$, and $N_{x y}$, which are then used for the computation of the matrix $\left[K_{\sigma}\right]$ : 
$\left[K_{\sigma}\right]=\iint[G]^{T}\left[\begin{array}{cc}N_{x} & N_{x y} \\ N_{x y} & N_{y}\end{array}\right][G] d x d y$

where $[G]$ is a matrix whose terms are obtained from the appropriate differentiation and ordering of the shape functions. The geometric stiffness matrix is usually computed for a set of reference loads, here denoted as $\{\mathrm{R}\}$ ref, which, for simplicity, are usually of unit magnitude. The geometric stiffness matrix corresponding to other load levels can be generally expressed as:

$$
\left[K_{\sigma}\right]=\lambda\left[K_{\sigma}\right]_{r e f} \text {, with }\{R\}=\lambda\{R\}_{\text {ref }}
$$

where $\lambda$ is a scalar multiplier. Letting $\{\delta \mathrm{D}\}$ be the buckling displacements relatively to $\{D\}_{\text {ref }}$, i.e. the displacement caused by the reference load, and given that the external loads do not change at a bifurcation point, the following relations hold:

$$
\left([K]+\lambda_{c r}\left[K_{\sigma}\right]_{r e f}\right)\{D\}_{r e f}=\lambda_{c r}\{R\}_{r e f}
$$

and

$$
\left([K]+\lambda_{c r}\left[K_{\sigma}\right]_{r e f}\right)\left\{D_{r e f}+\delta D\right\}=\lambda_{c r}\{R\}_{r e f}
$$

Subtracting equation (4) from equation (5) yields,

$$
\left([K]+\lambda_{c r}\left[K_{\sigma}\right]_{r e f}\right)\{\delta D\}=0
$$

which represents the condition at which the smallest root $\lambda_{c r}$ defines the smallest external load producing a bifurcation of the equilibrium state, i.e. more precisely:

$$
\{R\}_{c r}=\lambda_{c r}\{R\}_{r e f}
$$


The eigenvalue buckling solution required for the evaluation of $\lambda_{c r}$ from equation (7) is performed through Block Lanczos Extraction Method, which is particularly computationally efficient for models with a large number of constraint equations. The mode shapes $\{\delta D\}$ associated with the computed eigenvalues are then obtained through an expansion pass. In the current analysis, the mode shapes are extracted and observed to evaluate and identify local buckling mechanisms, which need to be differentiated from global buckling modes.

\subsection{Analytical evaluation of buckling loads}

Axially loaded deformable bodies may encounter instability under certain loading conditions, thus failing prematurely. Instability is a phenomenon of interest for thin shell structures, since the associated critical loads can be much smaller than the forces causing material collapse. The considered chiral honeycomb is composed of straight walls (ribs) that connect cylindrical shells (nodes), as shown in Figure 1. The buckling characteristics of the elementary components of a unit cell can be studied through analytical expressions found in the literature for plate and shell structures. The analytical results can be then used to validate the FE model, and to obtain insights regarding the overall buckling behavior of the considered assembly. The advantage of the developed FE model relies in its capability to predict global as well as local buckling modes, while generally analytical expressions for buckling of shells and plates only describe global buckling phenomena.

Each straight rib can be considered as a thin-plate structure constrained along the edges parallel to the loading direction. Results for this configuration are well known and can be found, for example, in [13]. The first buckling load $P_{c r}$ is given by:

$$
P_{c r}=\frac{K E_{s}}{\left(1-v_{s}^{2}\right)} \frac{t^{3}}{L}
$$

where $K$ is a numerical coefficient which depends on the boundary conditions applied along the edges parallel to the load. According to [8], $K$ is equal to 2.0 for simply 
supported edges, and $K=6.2$ for clamped boundaries. Given the particular conditions that characterize the rib/node connection in the chiral cell, $K$ has been selected as 5.2, through an iterative process based on a trial-and-error procedure. It is worth observing that this value lies between those corresponding to simply supported and fixed boundary conditions. From a physical point of view, this observation partially justifies the choice of $K=5.2$, as the rib of the chiral structure is modeled as rigidly connected to elastic, and therefore deformable, shells. Also in equation (8), $E_{s}$ and $v_{s}$ respectively are the Young's modulus and the Poisson's ratio of the material, $t$ is the thickness of the plate, and $L$ is the length of the rib measuring the distance between the two constrained edges.

The buckling behavior of axially loaded cylinders strongly depends on the cylinder's length-to-diameter ratio [13]. Short cylinders typically first show ring buckling modes, moderately long cylinders present diamond modes, while long cylinders basically behave as long columns. A convenient parameter to classify cylinders as "short" or "long" can be expressed as [15]:

$$
Z=\left(\frac{b}{r}\right)^{2}\left(\frac{r}{t}\right) \sqrt{\left(1-v_{s}^{2}\right)}
$$

where $b$ is the cylinder's axial length, $r$ is the cylinder's radius, and $t$ is the wall thickness. A cylinder is described as short if $Z<2.85$, whereas long cylinders are characterized by $Z>2.85$. The cylinders in the chiral honeycomb belong by far to the latter category, as demonstrated by the values listed in Table 1, which are computed for $b=0.14$ $\mathrm{m}, r=16 \mathrm{~mm}$ and for wall thickness $t$ varying between 0.1 and $1 \mathrm{~mm}$. The critical buckling stress for long cylinders can be expressed as [13]:

$$
\sigma_{c r}=\frac{E_{s}}{\sqrt{3\left(1-v_{s}^{2}\right)}} \frac{t}{r}
$$

and the corresponding critical axial load is given by: 


$$
N_{c r}=\frac{2 \pi E_{s} t^{2}}{\sqrt{3\left(1-v_{s}^{2}\right)}}
$$

The geometric parameters for the considered chiral cell are listed in Table 2. For this configuration, the critical load for a plate as given by equation (8) is always lower than that of the cylinder representing a node in the chiral assembly. Values of the critical stress computed for the configuration listed in Table 2 and wall thickness $t$ varying between 0.1 and $1 \mathrm{~mm}$ are listed in Table 3, where the critical stress given by equation (10) for shells is compared to that of the plate given by equation (8) normalized by the load-carrying area. These analytical results clearly show how the global buckling behavior of the chiral honeycomb cell is dominated by the collapse of the ribs. This conclusion agrees well with the results of the FE analysis presented in the following sections. In addition, the FE model provides information regarding local buckling limits and critical loads, which are also important if local plasticity or the insurgence of localized cracks is to be avoided.

\section{RESULTS AND DISCUSSION}

\subsection{Parametric Analysis}

The first step in surveying the chiral structure's response to simple compression under uniaxial-loading consists in observing the effects of various parameters that define the geometry of the unit cell. The influence of the wall thickness $t$ is initially considered. Results for varying rib length to node center ratios $L / R$ (see Figure 1), are then illustrated. The results obtained from the numerical analysis are compared to analytical models presented in section 2.7. The comparison is only made in terms of global buckling modes, as the theories referenced in section 2.7 are all limited to global behavior only. However, the sensitivity of local buckling to the cell configuration is also investigated, as in most applications it is desirable to avoid plastic deformations of a structure's honeycomb core. In the following sections, global buckling modes are also used to compare chiral 
honeycombs to traditional hexagonal honeycombs, in an effort to ensure a conservative comparison of the two honeycomb geometries.

The parameter values used in surveying the effects of varying wall thickness $t$ are again those summarized in Table 1 . Values of thickness varying between $1 \times 10^{-4}$ and $1 \times 10^{-3} \mathrm{~m}$, which are typical for metallic honeycombs, are considered. The lower limit of the range is selected on the basis of convergence limits encountered during the FE buckling analysis, while the upper value for $t$ is limited by the thin-wall assumption. The comparison of the global buckling loads predicted by the FE model and by equation (8) is given in Table 4, and it is shown graphically in Figure 7. An example of the corresponding buckling modes as predicted by the FE model is shown in Figure 8.a, which demonstrates how the mechanism of global buckling is governed by the collapse of the ribs. The plot of Figure 7 also presents the variation of the first or lower buckling mode predicted by the FE model. These loads correspond to local instabilities, and cannot be directly compared to the analytical results. For a rib thickness varying between $1 \times 10^{-4}$ and approximately $4 \times 10^{-4} \mathrm{~m}$, the local buckling load is on average $15 \%$ lower than the global-buckling load. A representative local buckling mode is shown in Figure 8.b.

The second step in the parametric analysis consists in observing the effects of the ratio $L / R$, with the objective of investigating the effect of rib length and distance between node centers on the buckling strength of the chiral assembly. The considered wall thickness $t$ is selected equal to $0.22 \mathrm{~mm}$, and the radius of the nodes $r$ is maintained constant and equal to the value listed in Table 1, so that the influence of the rib length $L$ on the buckling loads is isolated from that of the node radius. Given $r$, and imposing the $L / R$ ratio, one can calculate the angle $\beta$, which, from equation (1) can be found as:

$$
\beta=\cos ^{-1}\left(\frac{L}{R}\right)
$$

The corresponding values of $L$ and $R$ can be again found from equations (1). All other geometrical parameters and material properties are constant and equal to the values listed in Table 1. Figure 9 illustrates the variation in cell geometry as the ratio $L / R$ is increased 
from 0.74 to 0.998 . It is worth observing that the cells shown in Figure 9 have different overall dimensions, as varying $L / R$ corresponds to modifying the relative density of the assembly. The corresponding buckling loads are summarized in Table 5, and their variation with respect to $L / R$ is plotted in Figure 10. The critical buckling loads (global) obtained from the numerical analysis again closely agree with the values predicted by equation (8). The plot of Figure 10 shows that critical loads for local and global buckling converge as $L / R$ approaches 1 . This trend is confirmed by the corresponding buckling modes, where deformations corresponding to the lowest buckling load appear localized for low values of $L / R$, and tend to achieve global features as $L / R$ increases (Figure 11). The critical loads, hence, appear to be very dependent upon the ratio $L / R$. The possibility of obtaining configurations with significantly different properties while maintaining the overall geometry is one of the most attractive characteristics of this innovative honeycomb configuration. The results here presented show that simply changing the rib length or the distance between node centers, without changing material or wall thickness, dramatically increases the strength of the assembly when undergoing flatwise compression, and strongly modifies the deformation patterns associated with the onset of instability.

\subsection{Comparison with Traditional centro-symmetric structures}

The attractive characteristics of the chiral configuration as an alternative for applications in sandwich structures can be confirmed through a direct comparison with the compressive strength of traditional hexagonal and re-entrant honeycomb structures. The influence of the relative density on the buckling strength of chiral noncentrosymmetric configurations is investigated by varying the ratio $L / R$ in the range between 0.74 and 0.998 . As opposed to section 3.1, however, the parameters $r$ and $R$ are allowed to vary, while the rib length $L$ and the wall thickness $t$ are maintained constant and respectively equal to $0.07 \mathrm{~m}$ and $0.5 \mathrm{~mm}$. The considered unit cell for the hexagonal honeycomb is depicted in Figure 12. The wall thickness is selected equal to that of the chiral geometry, and the parameters $h$ and $l$ are also selected equal to the value of $L$ for 
the chiral structure. The internal angle $\theta_{h}$ is varied between $-30^{\circ}$ to $30^{\circ}$, in order to analyze the buckling strength of both hexagonal and re-entrant honeycombs. Examples of the geometries obtained by varying the parameter $\theta_{h}$ are shown in Figure 13. Auxetic honeycombs are here considered as they have demonstrated a higher compressive strength than traditional honeycombs as a result of their re-entrant configuration. The increased strength of auxetic honeycombs is documented in $[9,16]$. The load carrying area for hexagonal honeycombs, i.e. the area occupied by the considered unit cell on the $x y$ plane, is given by:

$$
A_{\text {hexagon }}=2 l^{2} \cos \theta_{h}\left[h / l+\sin \theta_{h}\right]
$$

While the area of the chiral cell shown in Figure 3 can be expressed as:

$$
A_{\text {chiral }}=4 \pi r^{2}+3 L^{2} \sin \theta
$$

The areas expressed in equations (13) and (14) are used for the computation of the relative density of the hexagonal and chiral topologies, which are respectively given by:

$$
\begin{aligned}
& \frac{\rho^{*}}{\rho_{s}}=\left[\frac{h / l+2}{2\left(h / l+\sin \theta_{h}\right) \cos \theta_{h}}\right] \frac{t}{l} \\
& \frac{\rho^{*}}{\rho_{s}}=\frac{t(4 \pi r+6 L)}{4 \pi r^{2}+3 L^{2} \sin \theta}
\end{aligned}
$$

The variation of the relative densities with respect to the parameters varied during the analysis, i.e. the internal angle $\theta_{h}$ for hexagonal honeycombs, and the $L / R$ ratio for the chiral structure, is shown in Figure 14. Figure 15 shows a direct comparison of the unit cell geometries for $L / R$ and $\theta_{h}$ at the limits of the considered ranges of variation. The buckling strength of the two cellular configurations is here compared in terms of the critical stress. Equations (15) and (16), and the plot of Figure 14 however show that the 
relative densities for the considered set of parameters are different. Hence, the critical stress obtained for each configuration is normalized with respect to the corresponding relative density in order to obtain a normalized equivalent strength, which accounts for the amount of material in each considered unit cell. In the FE analysis, the critical stress is obtained from the critical load resulting from the stability analysis, divided by the area defined in equation (14). The critical stress for the hexagonal honeycomb is not computed using Finite Elements, but through analytical formulas found in the literature $[9,16]$. These formulas are based on classical expressions for cellular materials [8], linearbuckling theories and equation (8) for plate-like structures. The expression of the critical stress for honeycombs with internal angle $\theta_{h}$ as found in $[9,16]$ is:

$$
\left(\sigma_{e l}^{*}\right)_{3}=\frac{2 E_{s}}{\left(1-v^{2}\right)} \frac{(h / l+2)}{\left(h / l+\sin \theta_{h}\right) \cos \theta_{h}}\left(\frac{t}{l}\right)^{3}
$$

Figure 16 presents a comparison of the normalized linear buckling stress for chiral, auxetic and hexagonal honeycombs. Both global and local buckling are presented for the chiral architecture to emphasize that also its linear local buckling load is higher per unit weight than the global critical load of auxetic and hexagonal configurations. It is important to observe how changing the chiral configuration, in this case through the variation of the $L / R$ ratio, produces strong changes in the normalized critical stress, to demonstrate once again the design flexibility of this innovative configuration. Hexagonal honeycombs, on the contrary, feature strength characteristics which only depend on the relative density of the assembly. This is clearly demonstrated by the corresponding curve shown in Figure 16, which shows that the strength of traditional honeycombs, if normalized with respect to the relative density, does not change with respect to changes in the internal angle of the layout. Another important observation is that the difference in buckling performance for the two considered geometries becomes very relevant as the ratio $L / R$ increases. These final observations are very important to highlight the potential benefits of applying the chiral geometry for the design of innovative sandwich configuration with higher flat-wise strength. 


\section{CONCLUSIONS}

The flat-wise compression of chiral honeycomb configurations is analyzed through a FE model and simple analytical relations. The analysis is based on linear buckling theory, which is usually accepted when considering the strength of honeycomb structures under flat-wise loads. The analytical study is based on formulas for thin-plate and long cylindrical shells found in the literature. The results from the FE model and the analytical formulas are compared with excellent agreement. The comparison is however limited to the global buckling loads, as local buckling phenomena for the considered structure can only be evaluated through the FE model. The numerical investigations hence provide information regarding both local and global buckling, conveying comprehensive information regarding the buckling behavior of this innovative cellular configuration. Global buckling of the honeycomb walls is typically considered as a limiting factor for flat-wise compressive loads and for the global structural integrity of sandwich structures with honeycomb core. Local buckling however should be considered if localized plasticity and subsequent damage is to be avoided.

The investigations presented in this paper are also completed by a comparison of the performance of chiral cellular structures with that of traditional hexagonal and re-entrant configurations. The comparison is performed by evaluating, for each configuration, the critical stress normalized by the corresponding relative density. The critical stress for hexagonal honeycombs is computed using an analytical formula that predicts linear global buckling of re-entrant and hexagonal honeycombs. The comparison shows that chiral topologies perform generally better in terms of both global and local, and that its local buckling loads are higher than those causing global collapse of traditional honeycombs. Furthermore, the strength of hexagonal structures is mostly influenced by their relative density, while substantial improvements can be obtained in chiral honeycombs even by keeping the relative density, and therefore the weight of the structure, constant. The presented results show the potentials of chiral structures as alternative configurations for sandwich components having great design flexibility and significantly superior flat-wise compression strength. 


\section{ACKNOWLEDGEMENTS}

This work is supported by the grant (W911NF0410141) from the Army Research Office, with Dr. Gary Anderson as program manager and technical monitor. The authors are grateful for the support and the invaluable technical inputs.

\section{REFERENCES}

[1] Lakes R. S., 1991 "Deformation mechanisms in negative Poisson's ratio materials: structural aspects". J. Mat. Sci. 26, pp. 2287-2292.

[2] Lakes R. S., 1987 "Foam structures with a negative Poisson's ratio", Science, 235, pp. 1038-1040.

[3] Alderson K. L., Alderson A. and Evans K. E., 1996 "The interpretation of the strain-dependent Poisson's ratio in auxetic polyethylene". Journal of Strain Analysis. 32(3), pp. 201-212.

[4] Scarpa F. and Tomlinson G., 2000 "Theoretical characteristics of the vibration of sandwich plates with in-plane negative Poisson's ratio values" Journal of Sound and Vibration 230(1), pp. 45-67.

[5] Herakovich C. T., 1984 "Composite laminates with negative through-thethickness Poisson's ratios". Journal of Composite Materials 18, pp. 447-455.

[6] Evans K. E., 1991 "Design of doubly curved sandwich panels with honeycomb cores" Computers and Structures, 17(2), 95-111.

[7] Prall, D., and Lakes, R. S., Properties of a Chiral honeycomb with Poisson's ratio of - 1, Int. J. of Mechanical Sciences, 39 (1996), 305-314.

[8] Gibson L. J. and Ashby M. F. Cellular solids: Structure and Properties, $2^{\text {nd }}$ Edition, 1997, Cambridge University Press, Cambridge, UK. 
[9] Zhang, J., and Ashby, M. F., The out-of-plane properties of honeycombs, Int. J. of Mechanical Sciences, 34 (1992), 475-489.

[10] Ansys, Inc, Theory Manual, Canonsburg, Pa.

[11] Papka, S. D., and Kyriakides, S., In-plane compressive response and Crushing of Honeycombs. Journal of the Mechanics and Physics of Solids, 41 (1994), 571 592.

[12] Cook, R. D., Malkus, D. S., Plesha, M. E., Witt, R. J., Concepts and Applications of Finite Element Analysis, $4^{\text {th }}$ Edition, 2001, Wiley, New York, NY.

[13] Timoshenko, S. P., and Gere, J. M., Theory of Elastic Stability, $2^{\text {nd }}$ Edition, 1961, McGraw-Hill, Tokyo.

[14] Farshad, M., Design and Analysis of Shell Structures, 1992, Kluwer Academic, Boston.

[15] Narayanan, R., Shell Structures: Stability and Strength, 1985, Elsevier Applied Science, London, UK.

[16] Scarpa F., Buriesci G., Smith F.C., Chambers B., "Mechanical and Electromagnetic Behaviour of Auxetic Honeycomb Structures" The Aeronautical Journal, 107 (2003), 175-183. 


\section{LIST OF TABLES}

Table 1: Variation of the $Z$ parameter versus wall thickness $t$ for the cylinder of the chiral assembly (cylinder radius $r=16 \mathrm{~mm}$ ).

Table 2: Geometric parameters and material properties of the chiral-honeycomb cell used for investigating the effects of wall thickness on the critical load.

Table 3: Analytical critical buckling stress for cylinder and ribs of the chiral cell. Variation with respect to the wall thickness.

Table 4: First global buckling load: comparison between analytical and FE predictions for varying wall thickness $t$.

Table 5: First global buckling load: comparison between analytical and FE predictions for varying $L / R$ ratio.

\section{LIST OF FIGURES}

Figure 1: A chiral cellular structure (a) and details of its main geometrical parameters (b) (from [7]).

Figure 2: Flat-wise compression of a chiral structure.

Figure 3. Unit cell of the chiral honeycomb.

Figure 4: Lines divisions in the unit cell (a) and considered FE mesh (b).

Figure 5: Periodic conditions imposed on the end nodes.

Figure 6: Schematic of loading configuration and considered boundary conditions.

Figure 7: Variation of global and local buckling loads for increasing wall thickness $t$.

Figure 8: First global (a) and local (b) buckling modes for the chiral cell.

Figure 9: Chiral unit cell with $r=0.016 \mathrm{~m}$, and $L / R$ equal to 0.997 (a), 0.985 (b), 0.933 (c), 0.85 (d), and 0.74 (e).

Figure 10: Variation of global and local buckling loads for increasing $L / R$ ratios.

Figure 11: Buckled chiral unit cell: $L / R=0.74$ (a), $L / R=0.995$ (b).

Figure 12: Geometric parameters of a typical hexagonal honeycomb.

Figure 13: Hexagonal unit-cells with $\theta_{h}=-25^{\circ}$ (a), $\theta_{h}=-10^{\circ}$ (b), $\theta_{h}=0^{\circ}$ (c), $\theta_{h}=10^{\circ}$ (d), $\theta_{h}=30^{\circ}(\mathrm{e})$.

Figure 14: Hexagonal and Chiral honeycomb relative densities.

Figure 15: Comparisons of hexagonal and chiral honeycomb cells.

Figure 16: Chiral and hexagonal honeycombs buckling stress normalized by relative density. 


\section{TABLES}

Table 1: Variation of the $Z$ parameter versus wall thickness $t$ for the cylinder of the chiral assembly (cylinder radius $r=16 \mathrm{~mm}$ )

\begin{tabular}{|c|c|}
\hline $\boldsymbol{t}[\mathbf{m m}]$ & $\boldsymbol{Z}$ \\
\hline \hline 0.1 & 11564 \\
\hline 0.19 & 6086 \\
\hline 0.28 & 4130 \\
\hline 0.37 & 3125 \\
\hline 0.46 & 2514 \\
\hline 0.55 & 2103 \\
\hline 0.64 & 1807 \\
\hline 0.73 & 1584 \\
\hline 0.82 & 1410 \\
\hline 0.91 & 1271 \\
\hline 1 & 1156 \\
\hline
\end{tabular}

Table 2: Geometric parameters and material properties of the chiral-honeycomb cell used for investigating the effects of wall thickness on the critical load.

\begin{tabular}{|c|c|}
\hline$L$ & $0.0832 \mathrm{~m}$ \\
\hline$\theta$ & $30^{\circ}$ \\
\hline$r$ & $0.016 \mathrm{~m}$ \\
\hline$R$ & $0.0892 \mathrm{~m}$ \\
\hline$b$ & $0.14 \mathrm{~m}$ \\
\hline$E_{s}$ & $2.2 \times 10^{9} \mathrm{~Pa}$ \\
\hline$v_{s}$ & 0.33 \\
\hline
\end{tabular}


Table 3: Analytical critical buckling stress for cylinder and ribs of the chiral cell. Variation with respect to the wall thickness.

\begin{tabular}{|c|c|c|}
\hline $\boldsymbol{t}[\mathbf{m m}]$ & $\begin{array}{c}\text { Cylinder } \\
\boldsymbol{\sigma}_{\boldsymbol{c r}}[\mathbf{K P a}]\end{array}$ & $\begin{array}{c}\text { Plate } \\
\boldsymbol{\sigma}_{\boldsymbol{c r}}[\mathbf{K P a}]\end{array}$ \\
\hline \hline 0.1 & 8409.7 & 17.7 \\
\hline 0.19 & 15978.4 & 63.8 \\
\hline 0.28 & 23547.1 & 138.6 \\
\hline 0.37 & 31115.8 & 242.0 \\
\hline 0.46 & 38684.5 & 374.0 \\
\hline 0.55 & 46253.2 & 534.7 \\
\hline 0.64 & 53821.9 & 724.0 \\
\hline 0.73 & 61390.6 & 941.9 \\
\hline 0.82 & 68959.3 & 1188.5 \\
\hline 0.91 & 76528.0 & 1463.7 \\
\hline 1 & 84096.7 & 1767.6 \\
\hline
\end{tabular}

Table 4: First global buckling load: comparison between analytical and FE predictions for varying wall thickness $t$

\begin{tabular}{|c|c|c|}
\hline $\mathbf{t}[\mathbf{m m}]$ & $\begin{array}{c}\text { Pcr [N] } \\
\text { (Analytical) }\end{array}$ & $\begin{array}{c}\text { Pcr [N] } \\
\text { (FE) }\end{array}$ \\
\hline \hline 0.1 & 1.9 & 1.9 \\
\hline 0.19 & 12.7 & 13.1 \\
\hline 0.28 & 40.6 & 41.8 \\
\hline 0.37 & 93.8 & 95.7 \\
\hline 0.46 & 180.2 & 182.9 \\
\hline 0.55 & 308.1 & 311.2 \\
\hline 0.64 & 485.4 & 488.5 \\
\hline 0.73 & 720.3 & 722.9 \\
\hline 0.82 & 1020.9 & 1021.9 \\
\hline 0.91 & 1395.3 & 1393.2 \\
\hline 1 & 1851.6 & 1844.4 \\
\hline
\end{tabular}


Table 5: First global buckling load: comparison between analytical and FE predictions for varying $L / R$ ratio.

\begin{tabular}{|c|c|c|c|c|c|}
\hline $\boldsymbol{L} / \boldsymbol{R}$ & $\begin{array}{c}\boldsymbol{\beta} \\
{[\mathbf{d e g}]}\end{array}$ & $\begin{array}{c}\boldsymbol{L} \\
{[\mathbf{m}]}\end{array}$ & $\begin{array}{c}\boldsymbol{R} \\
{[\mathbf{m}]}\end{array}$ & $\begin{array}{c}\boldsymbol{P c r}[\mathbf{N}] \\
\text { (Analytical) }\end{array}$ & $\begin{array}{c}\boldsymbol{P c r}[\mathbf{N}] \\
(\mathbf{F E})\end{array}$ \\
\hline \hline 0.74 & 42.3 & 0.04 & 0.05 & 46.6 & 44.4 \\
\hline 0.75 & 41.4 & 0.04 & 0.05 & 44.8 & 42.9 \\
\hline 0.77 & 39.6 & 0.04 & 0.05 & 43 & 41.4 \\
\hline 0.78 & 38.7 & 0.04 & 0.05 & 41.3 & 39.9 \\
\hline 0.79 & 37.8 & 0.04 & 0.05 & 39.6 & 38.4 \\
\hline 0.8 & 36.9 & 0.04 & 0.05 & 37.8 & 36.9 \\
\hline 0.82 & 34.9 & 0.05 & 0.06 & 36.1 & 35.3 \\
\hline 0.83 & 33.9 & 0.05 & 0.06 & 34.4 & 33.8 \\
\hline 0.84 & 32.9 & 0.05 & 0.06 & 32.7 & 32.3 \\
\hline 0.86 & 30.7 & 0.05 & 0.06 & 30.9 & 30.7 \\
\hline 0.87 & 29.5 & 0.06 & 0.06 & 29.2 & 29.2 \\
\hline 0.88 & 28.4 & 0.06 & 0.07 & 27.4 & 27.5 \\
\hline 0.89 & 27.1 & 0.06 & 0.07 & 25.6 & 25.9 \\
\hline 0.91 & 24.5 & 0.07 & 0.08 & 23.7 & 24.1 \\
\hline 0.92 & 23.1 & 0.08 & 0.08 & 21.7 & 22.3 \\
\hline 0.93 & 21.6 & 0.08 & 0.09 & 19.7 & 20.3 \\
\hline 0.95 & 18.2 & 0.09 & 0.1 & 17.5 & 18.2 \\
\hline 0.96 & 16.3 & 0.11 & 0.11 & 15.1 & 15.9 \\
\hline 0.97 & 14.1 & 0.13 & 0.14 & 12.3 & 12.9 \\
\hline 0.99 & 8.1 & 0.18 & 0.19 & 8.9 & 9.6 \\
\hline 0.998 & 3.6 & 0.51 & 0.51 & 3.2 & 2.7 \\
\hline
\end{tabular}




\section{FIGURES}

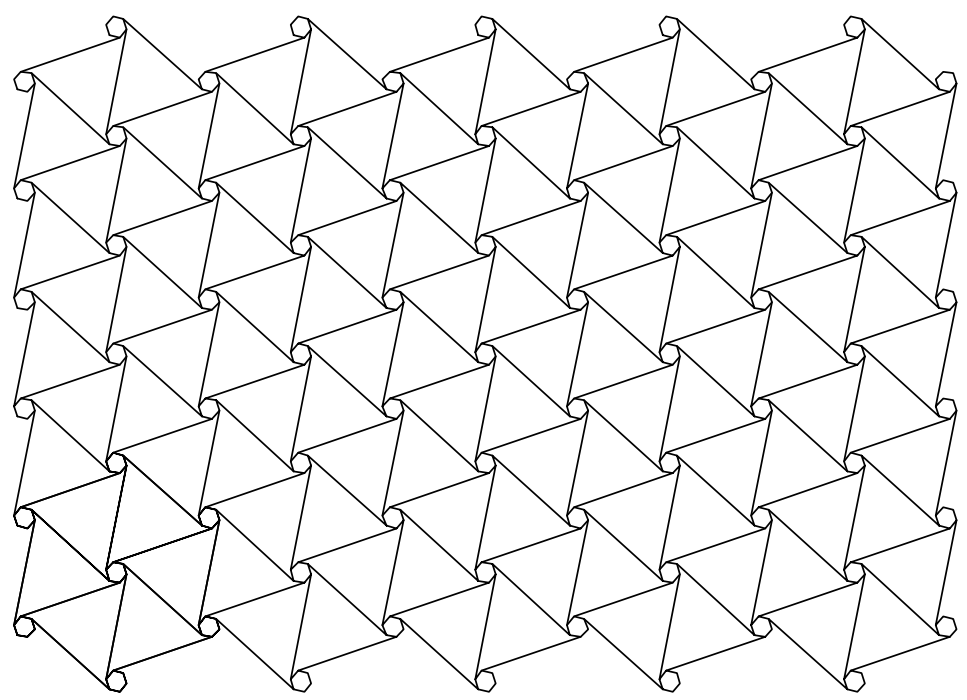

(a)

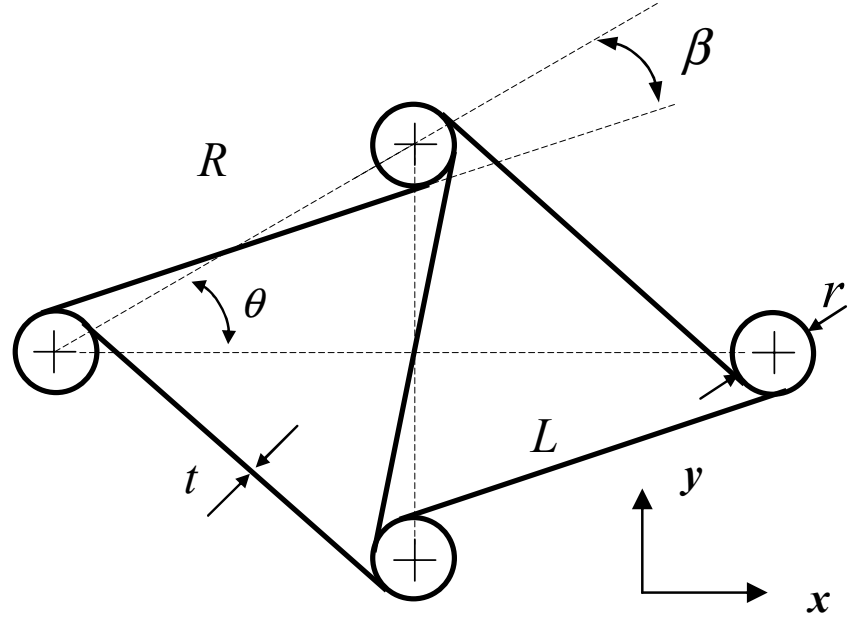

(b)

Figure 1: A chiral cellular structure (a) and details of its main geometrical parameters (b), (from [7]). 


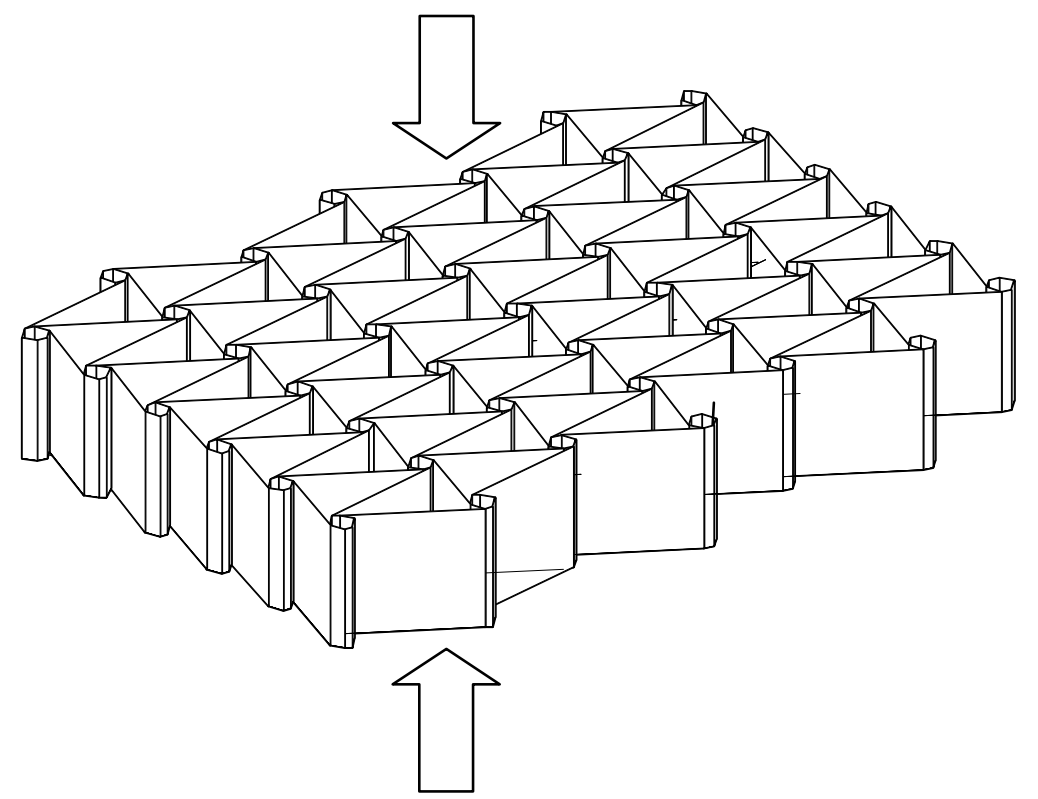

Figure 2: Flat-wise compression of a chiral structure. 


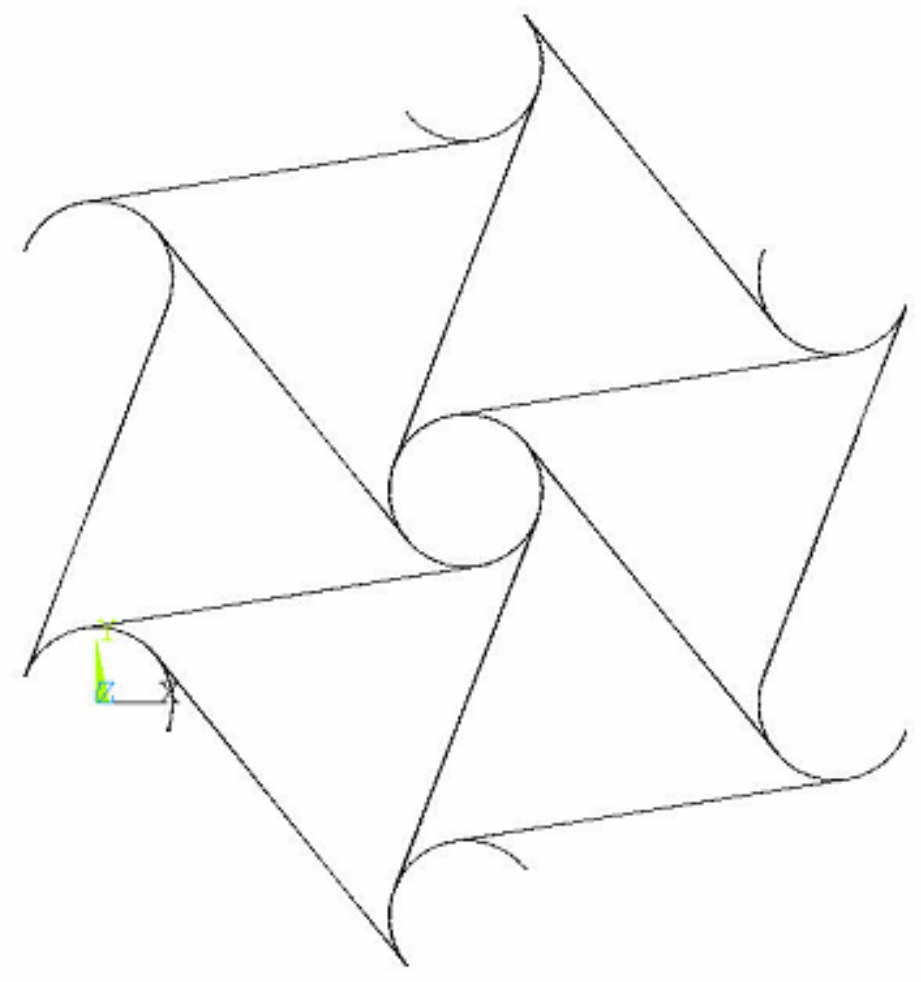

Figure 3. Unit cell of the chiral honeycomb. 


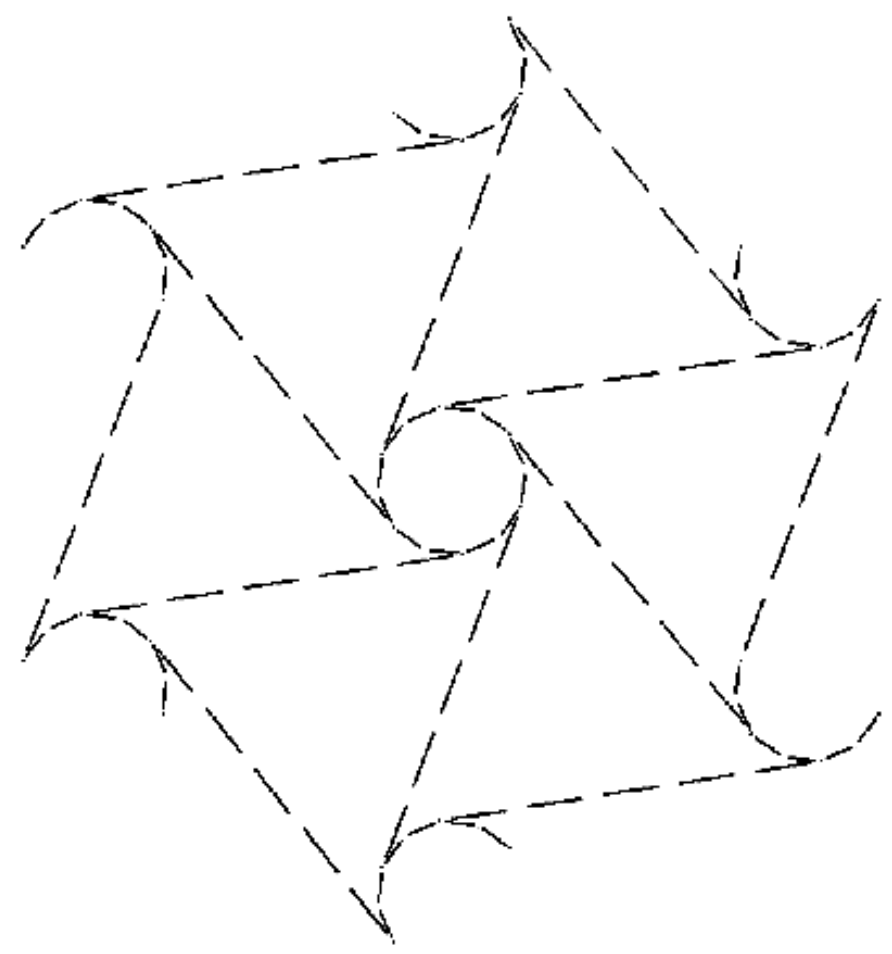

(a)

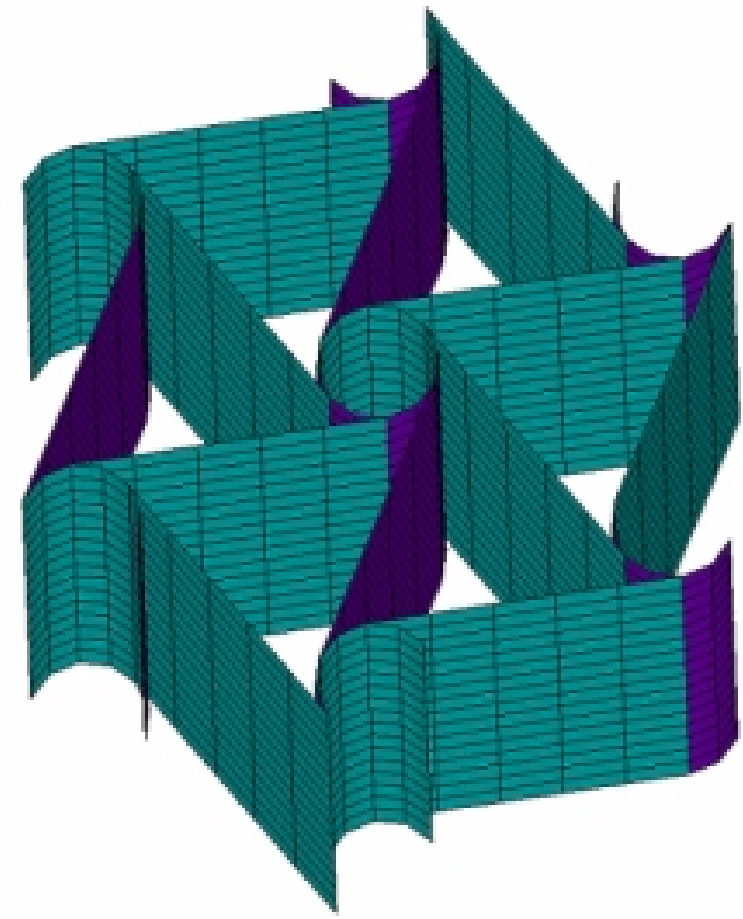

(b)

Figure 4: Lines divisions in the unit cell (a) and considered FE mesh (b). 


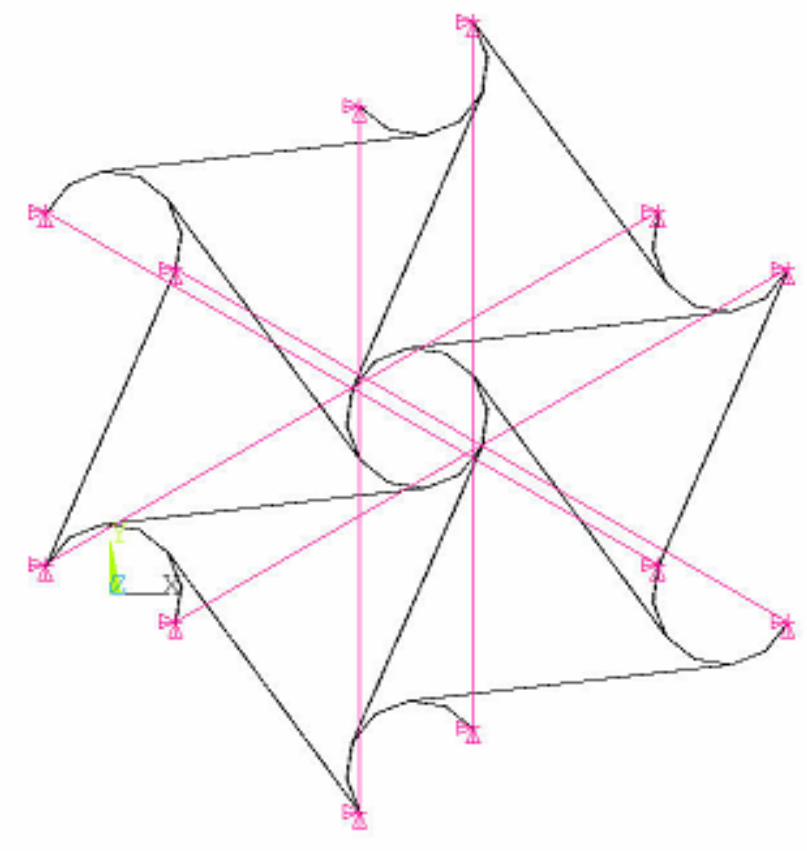

Figure 5: Periodic conditions imposed on the end nodes. 


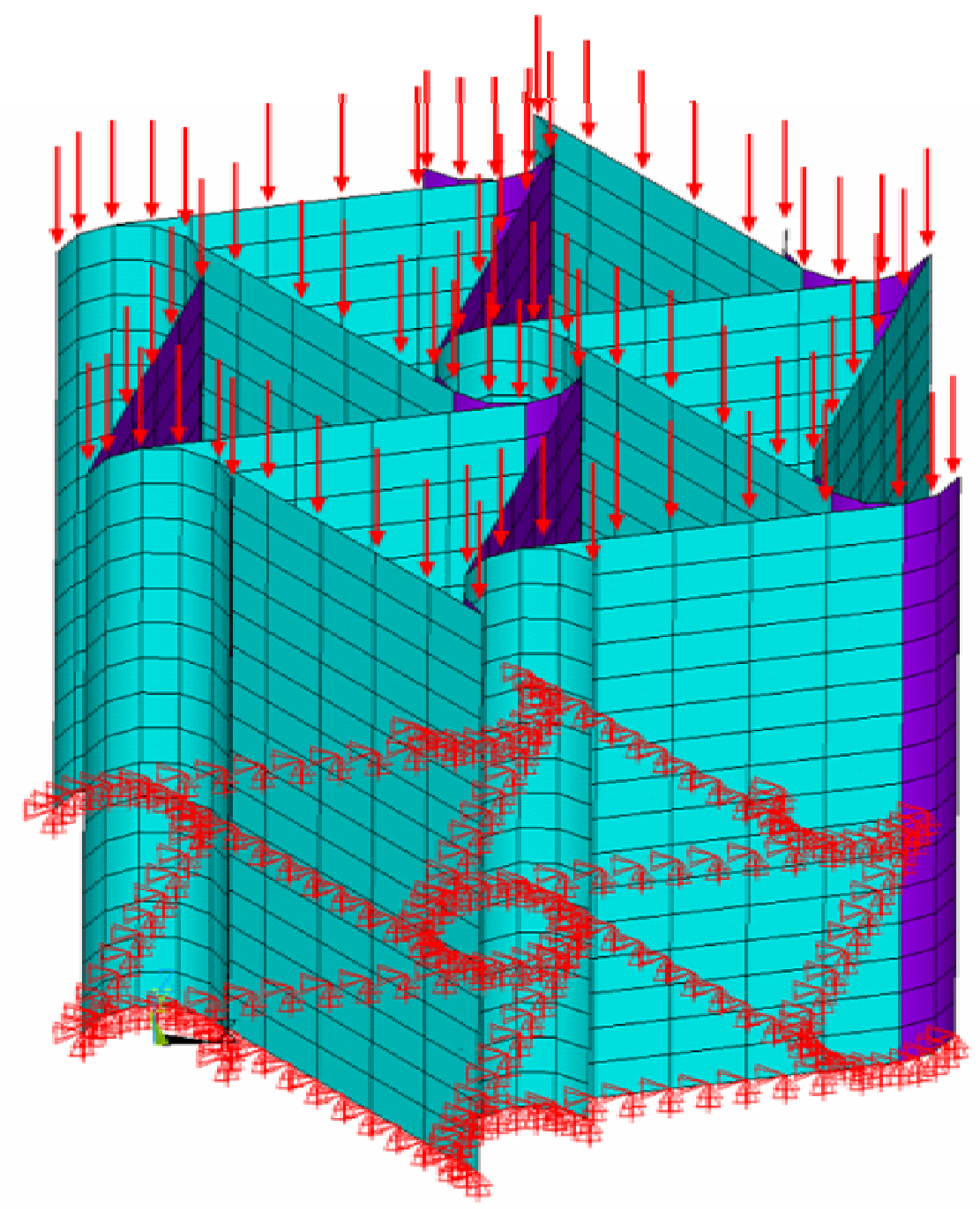

Figure 6: Schematic of loading configuration and considered boundary conditions. 


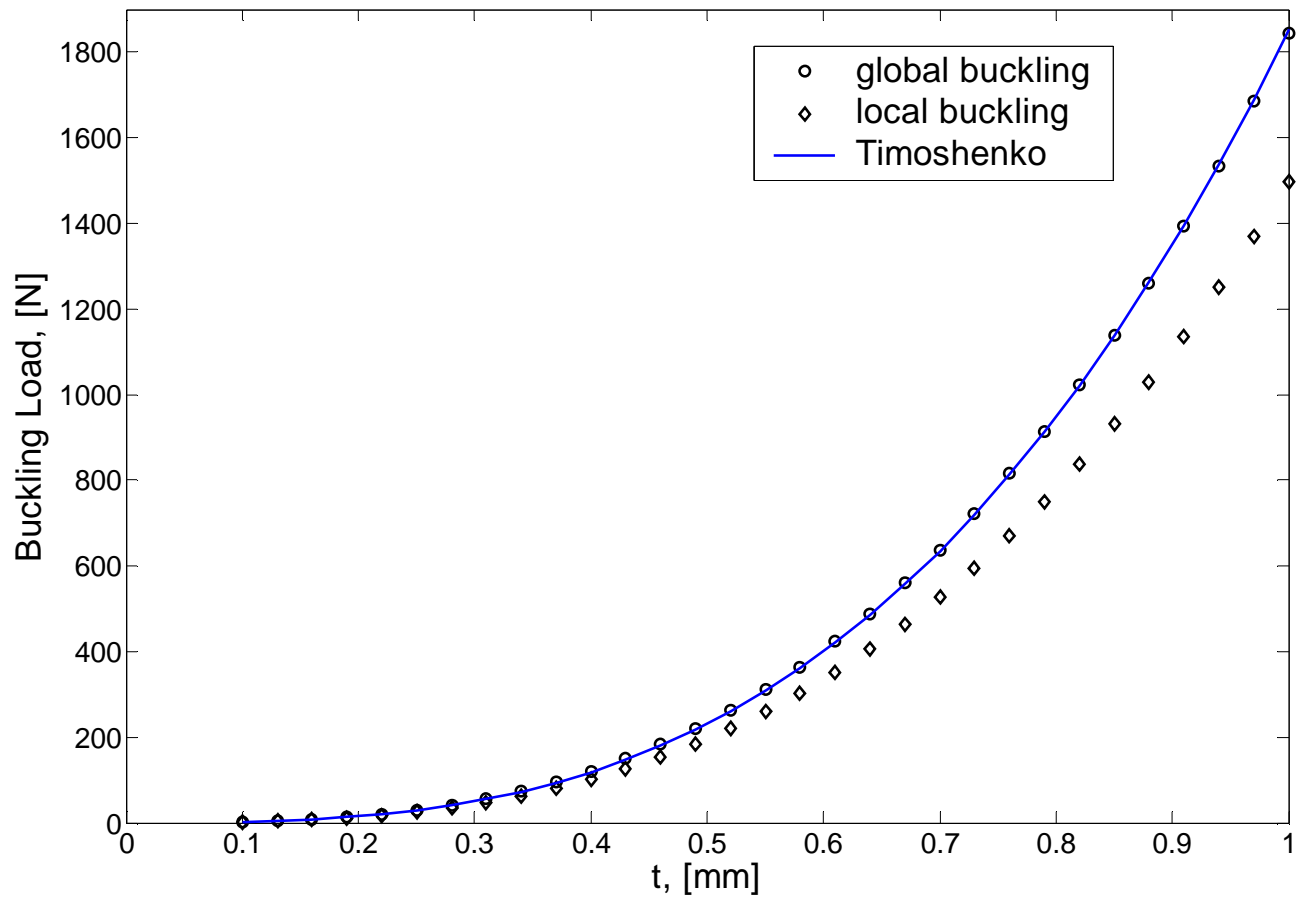

Figure 7: Variation of global and local buckling loads for increasing wall thickness $t$. 


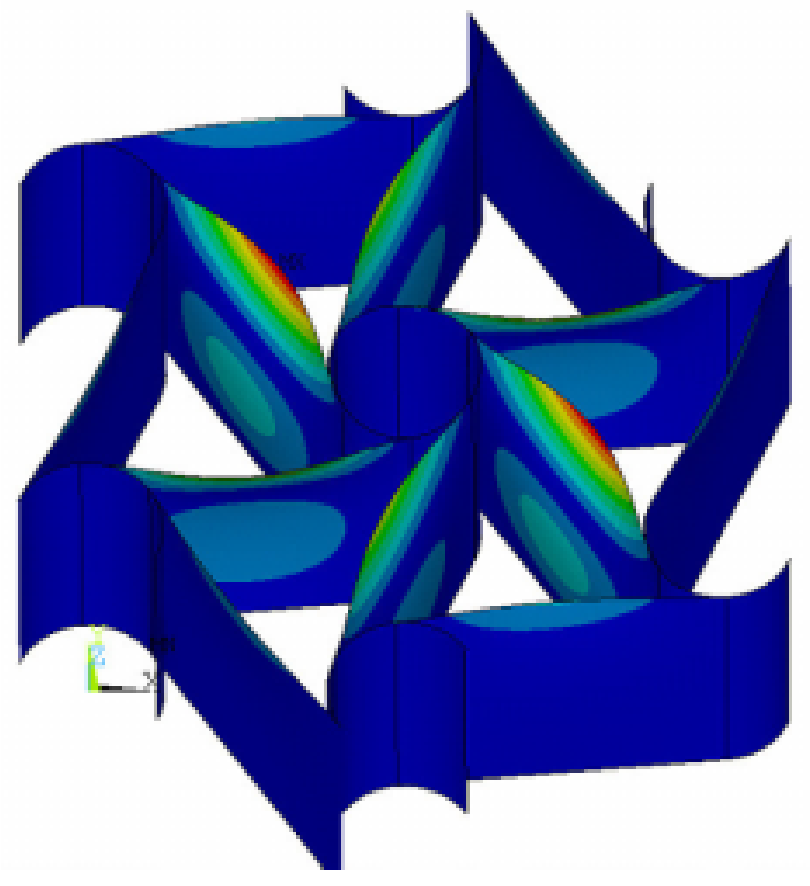

(a)

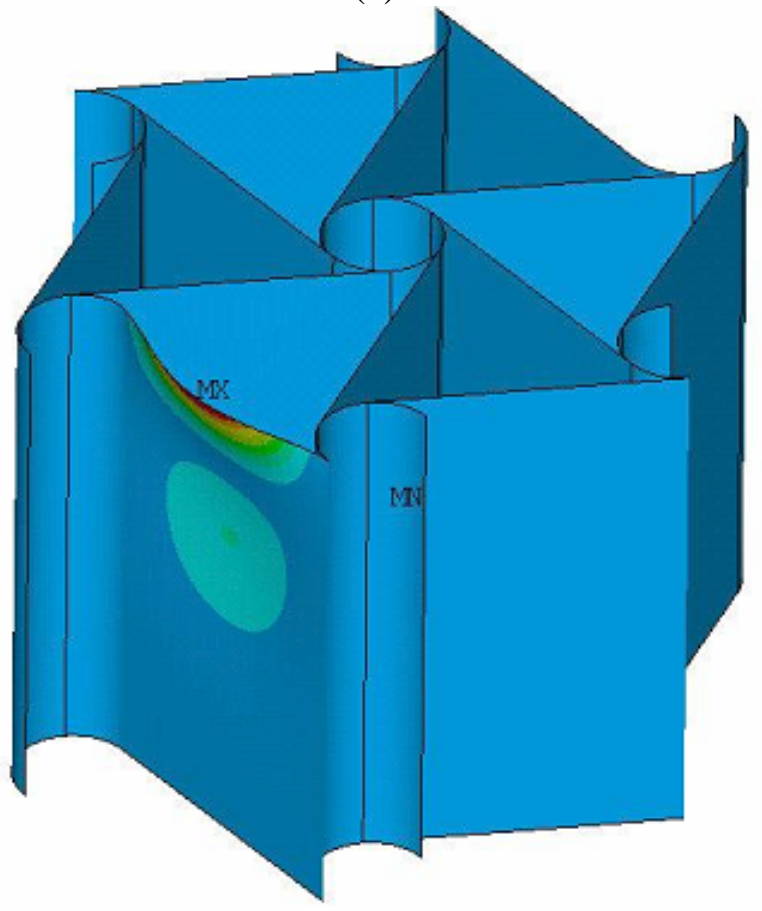

(b)

Figure 8: First global (a) and local (b) buckling modes for the chiral. 


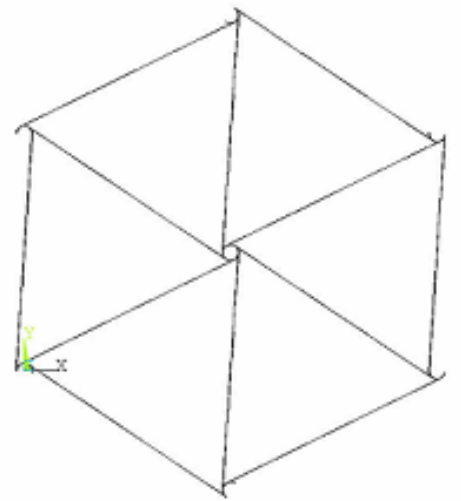

(a)

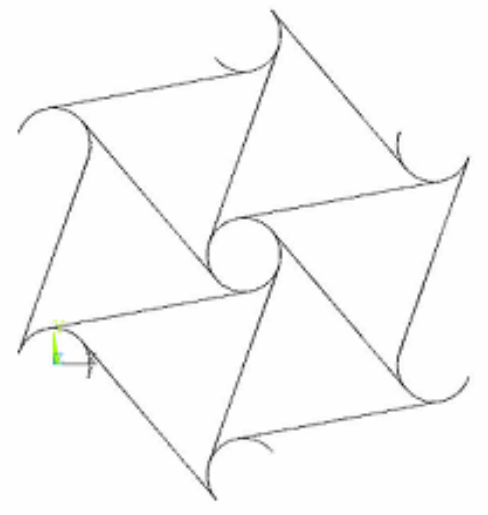

(c)

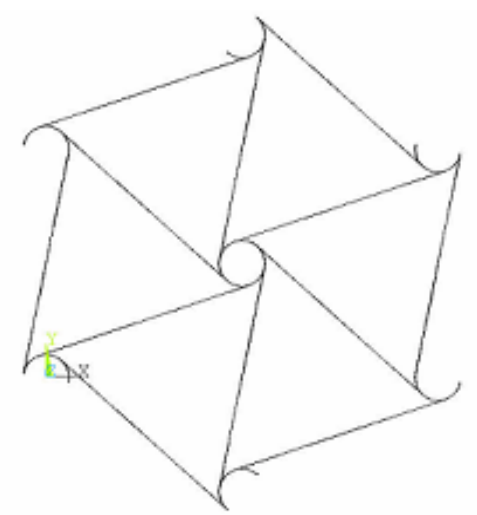

(b)

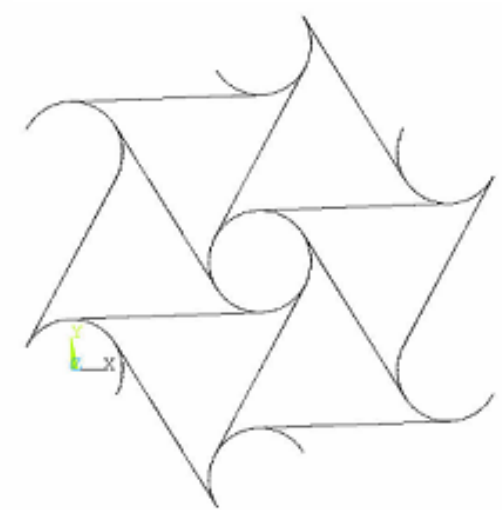

(d)

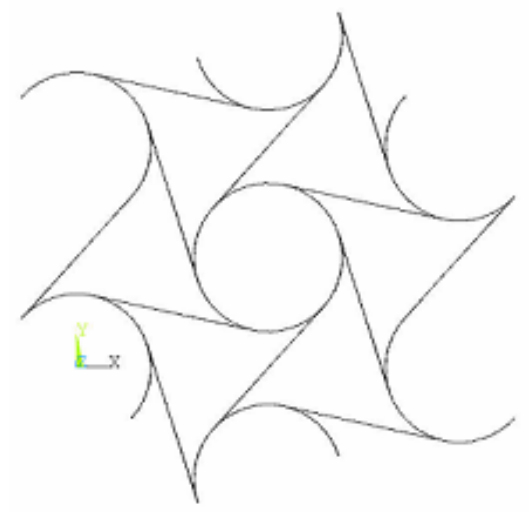

(e)

Figure 9: Chiral unit-cell with $r=0.016 \mathrm{~m}$, and $L / R$ equal to 0.997 (a), 0.985 (b), 0.933 (c), 0.85 (d), and 0.74 (e). 


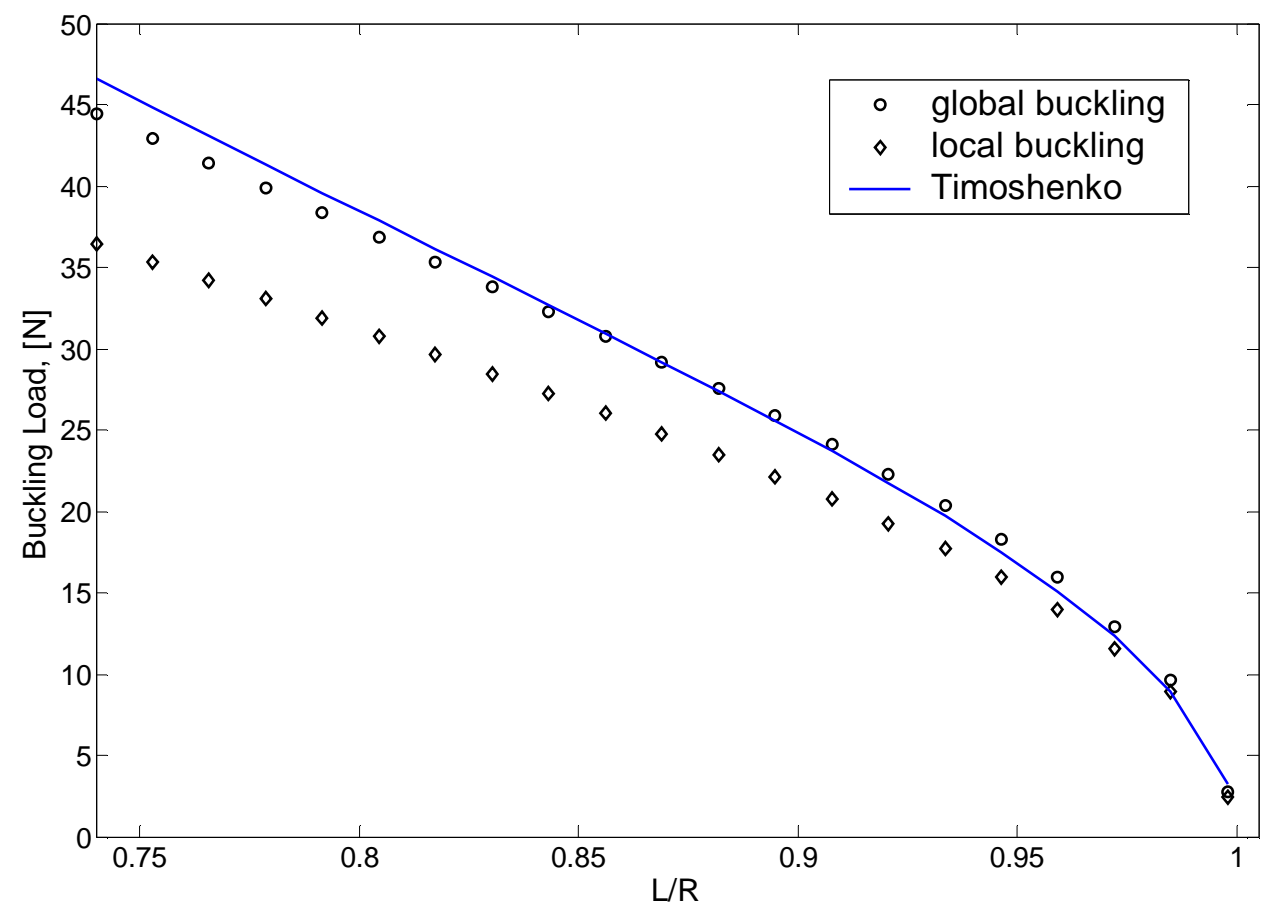

Figure 10: Variation of global and local buckling loads for increasing $L / R$ ratios. 


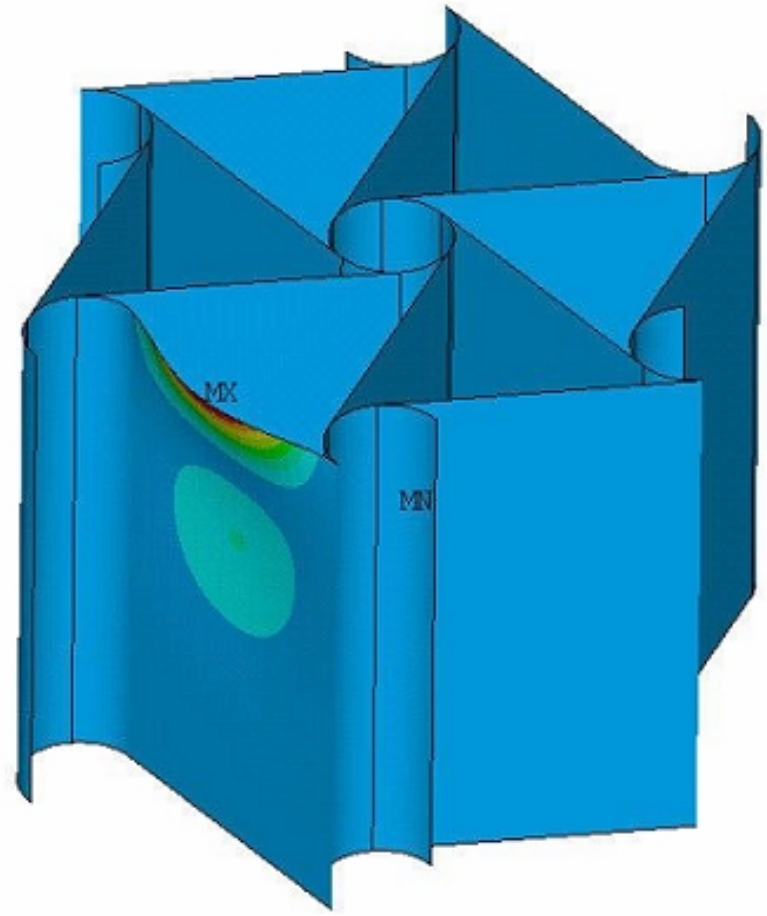

(a)

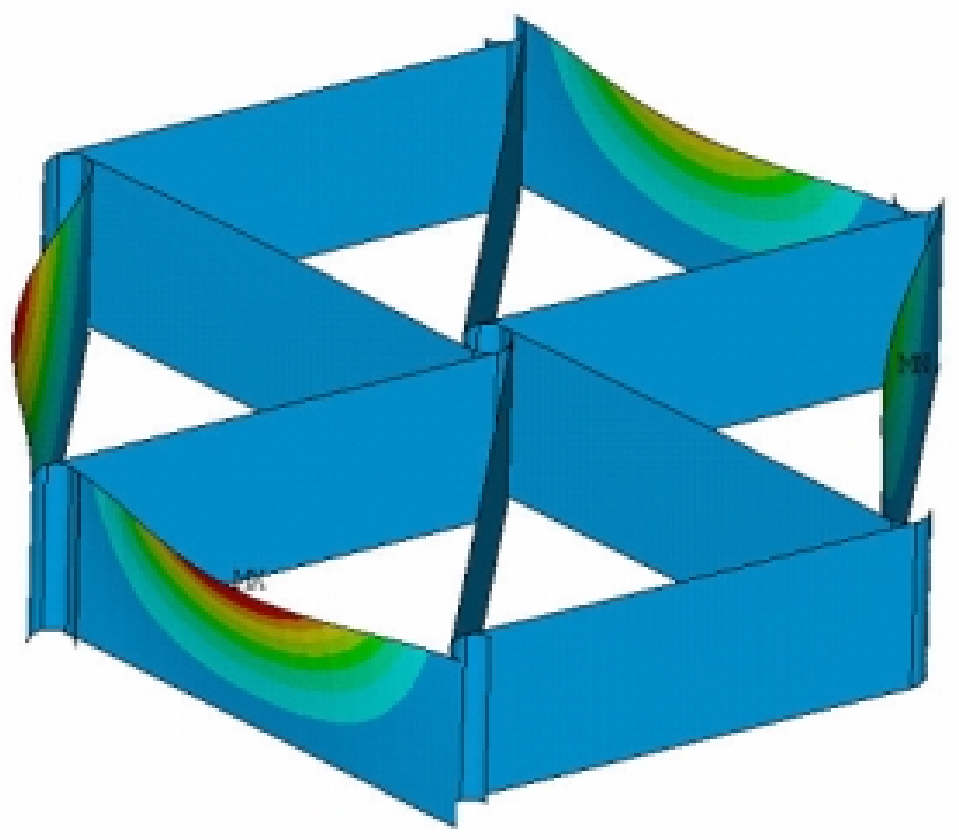

(b)

Figure 11: Buckled chiral unit cell: $L / R=0.74$ (a), $L / R=0.995$ (b). 


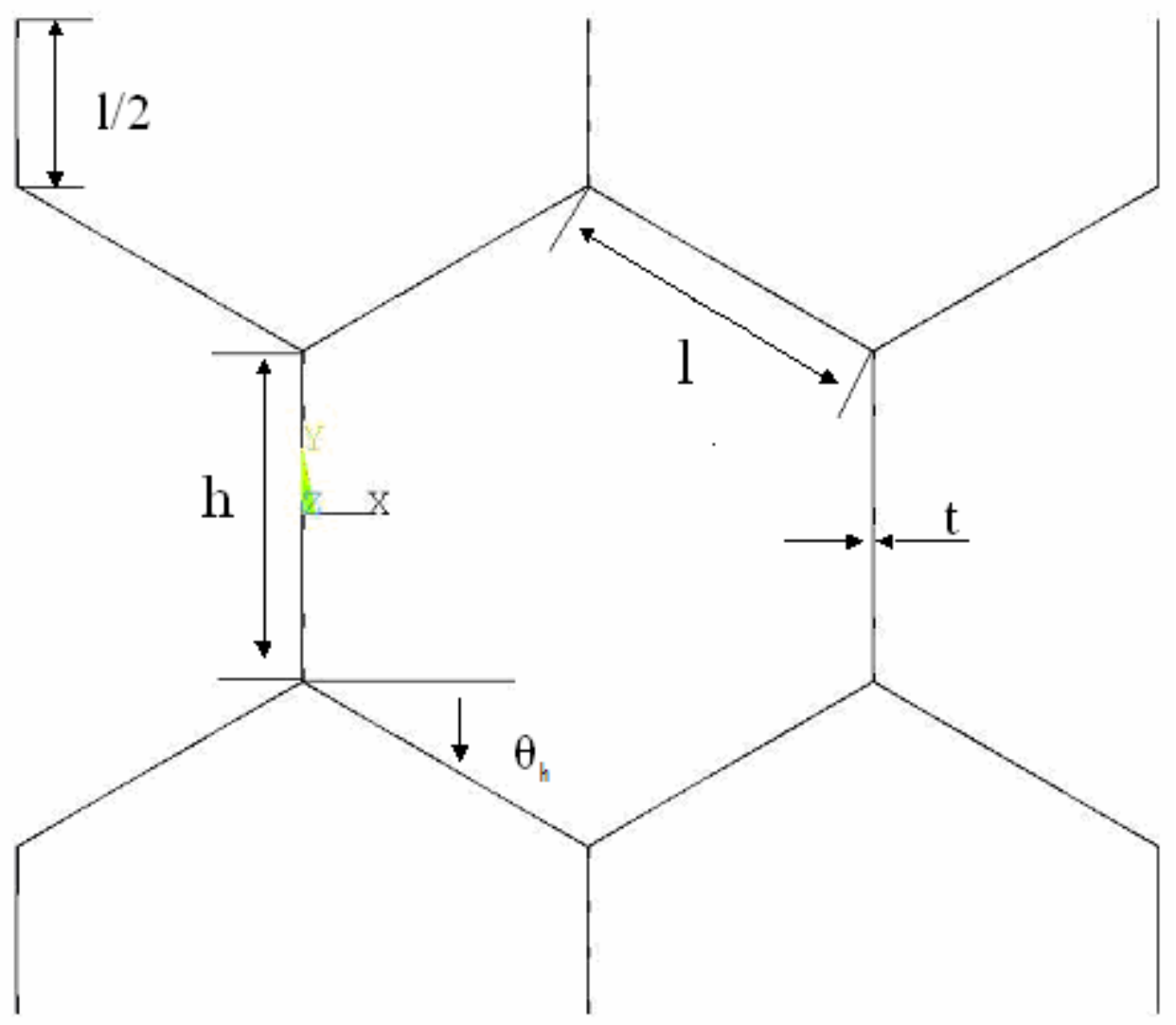

Figure 12: Geometric parameters of a typical hexagonal honeycomb. 


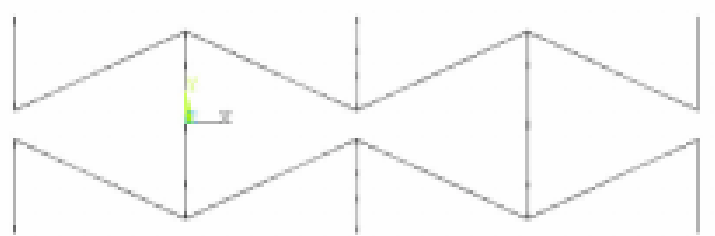

(a)

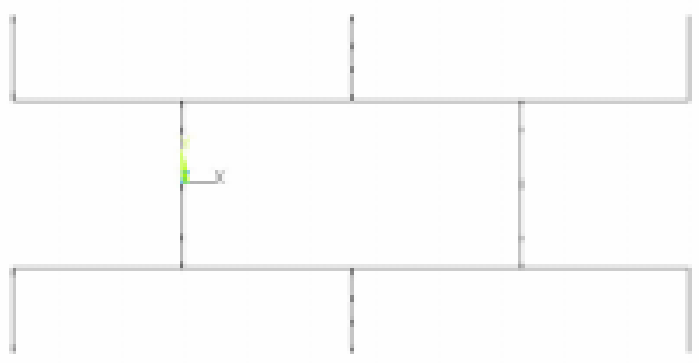

(c)

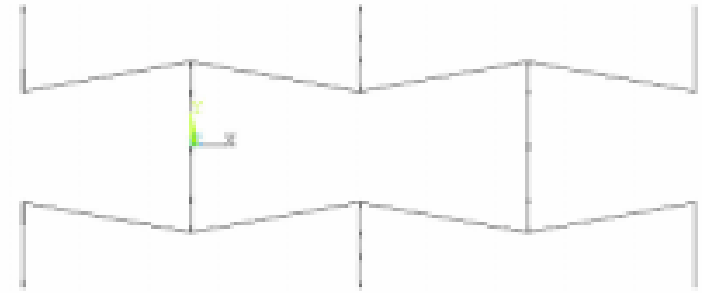

(b)

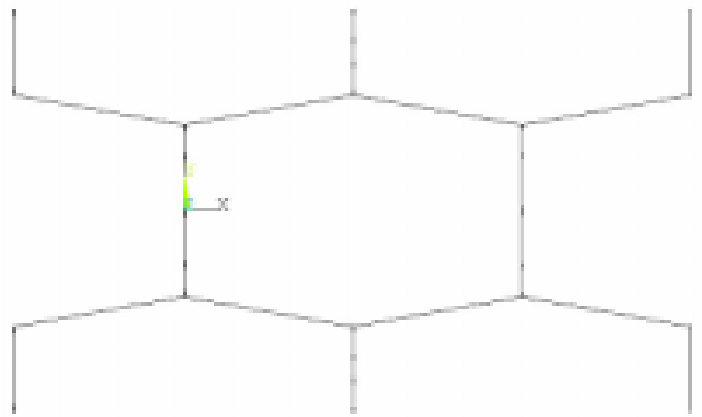

(d)

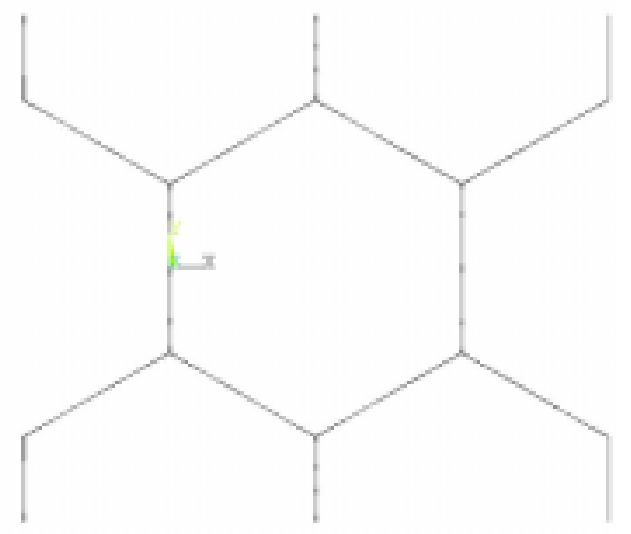

(e)

Figure 13: Hexagonal unit cells with $\theta_{h}=-25^{\circ}(\mathrm{a}), \theta_{h}=-10^{\circ}(\mathrm{b}), \theta_{h}=0^{\circ}(\mathrm{c}), \theta_{h}=10^{\circ}$ (d), $\theta_{h}=30^{\circ}(\mathrm{e})$. 


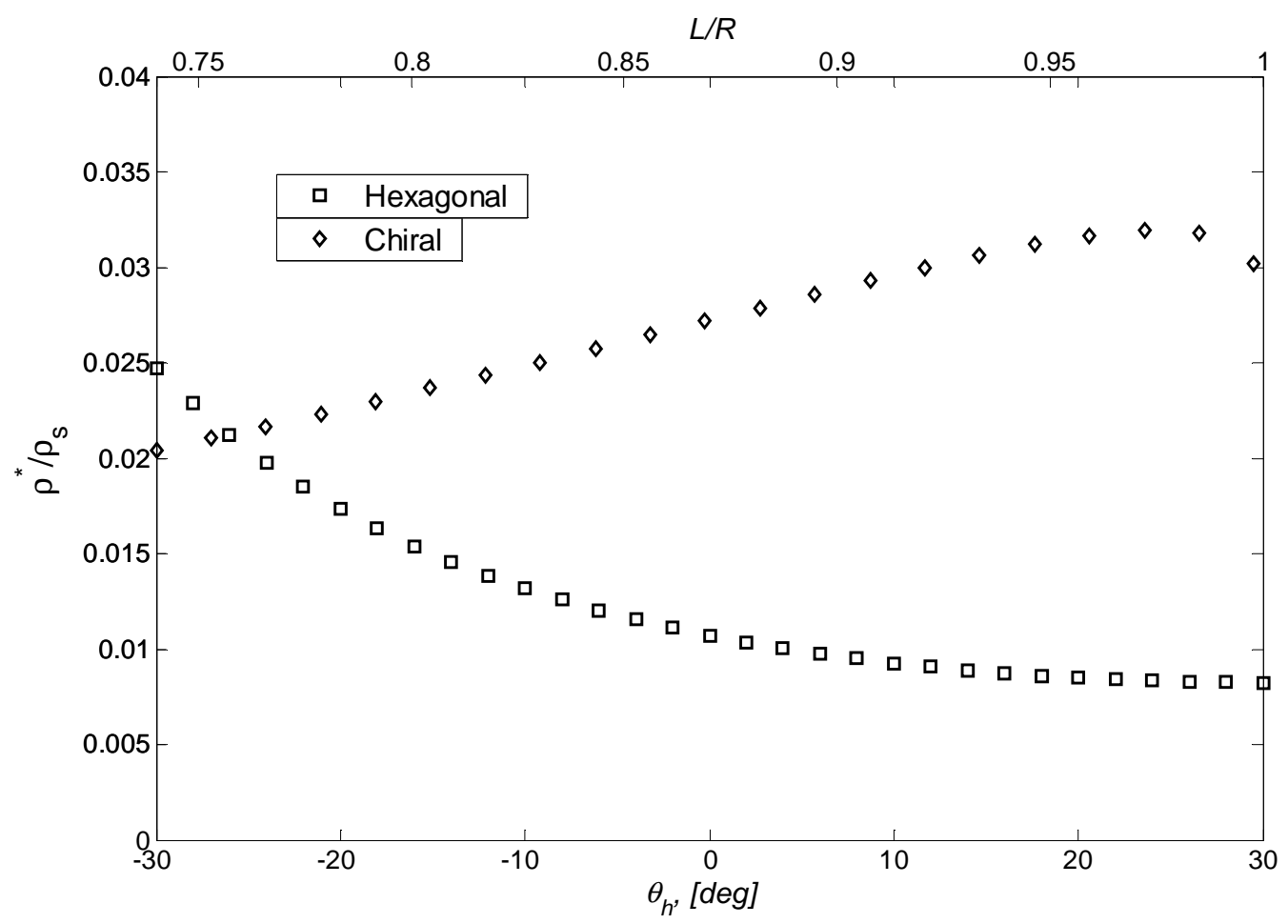

Figure 14: Hexagonal and chiral honeycomb relative densities. 

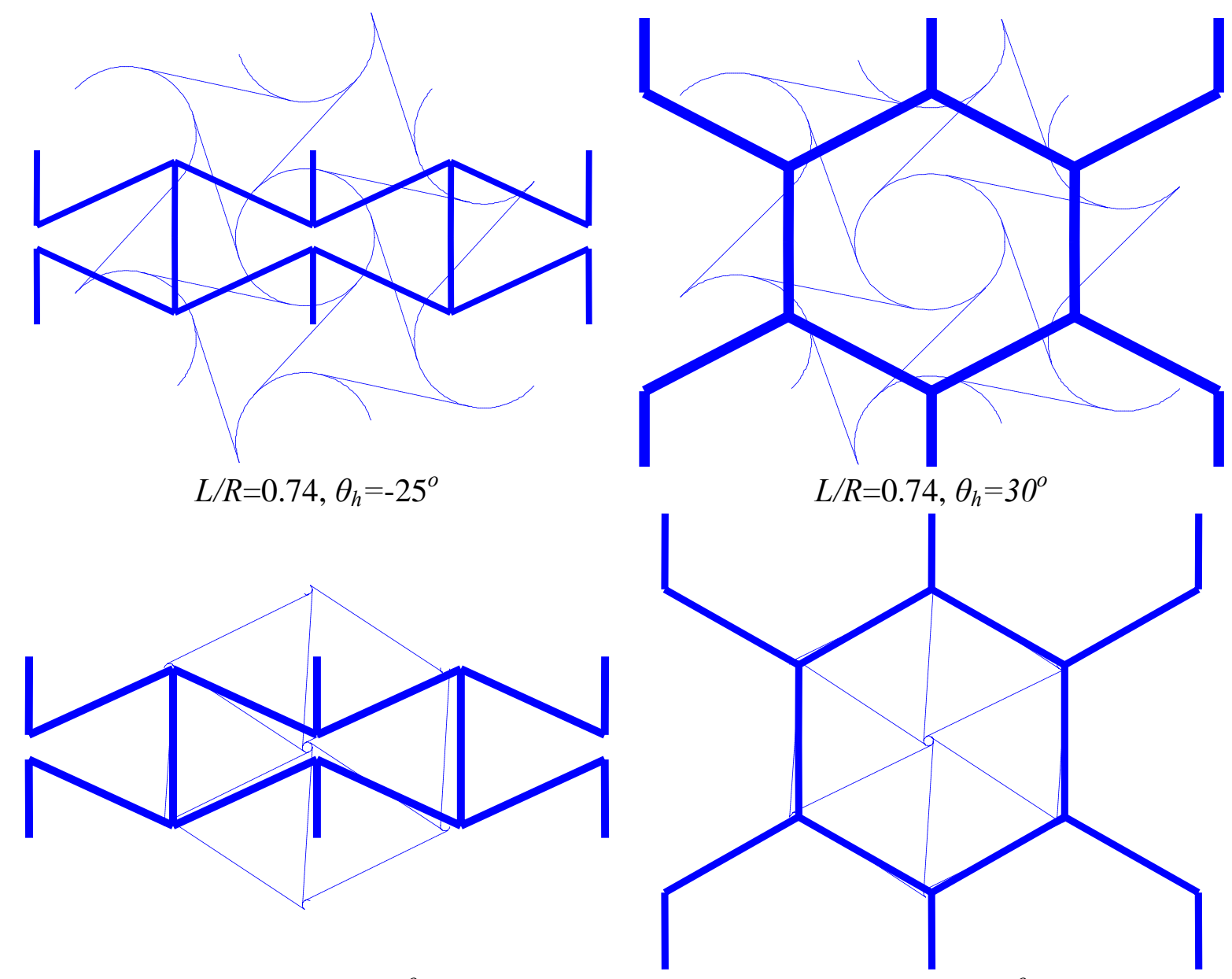

$$
L / R=1, \theta_{h}=-25^{\circ}
$$

$$
L / R=1, \theta_{h}=30^{\circ}
$$

Figure 15: Comparisons of hexagonal and chiral honeycomb cells. 


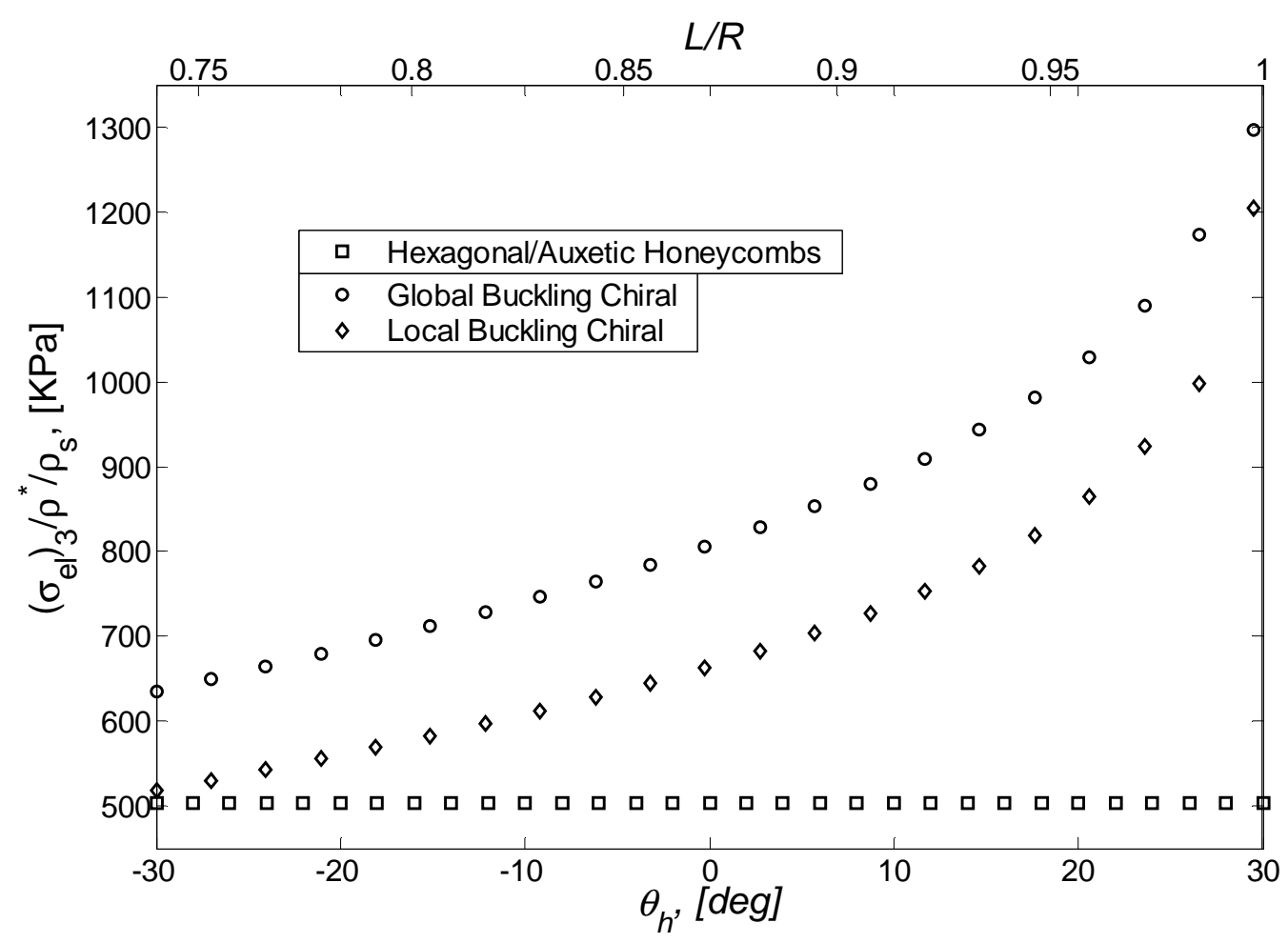

Figure 16: Chiral and hexagonal honeycombs buckling stress normalized by relative density. 